\title{
No more walls
}

\author{
Scientists can play an important role in the global society if they look beyond the walls of \\ traditional disciplines.
}

The ninth of November marks a few events in German history: the beginning of a short democratic period in 1918, devastating anti-Semitic pogroms in 1938 and the fall of the Berlin Wall in 1989. Since 2009, the Falling Walls Foundation - a charity supported by the German Federal Ministry of Education and Research, the Helmholtz Association, the Robert Bosch Stiftung, the Berlin Senate Department for Economics, Technology and Research, and various academic institutions, companies, non-governmental organizations and individuals - has commemorated this date through the Falling Walls conference, which aims to "connect people and their ideas across boundaries and disciplines" and encourages all "to tear down further walls in science and our global society" (http://www.falling-walls.com).

It is an unusual science conference in that it brings in experts from diverse fields and provides a platform for participants to share their knowledge and exchange ideas, all with the hope of inspiring those who attend and creating new cooperation between unlikely parties. This year heard from experts in nanotechnology, artificial intelligence and cultural anthropology. And knowledge sharing is made possible through the Brain Dating Lounge, which combines online social networking with an offline face-to-face 'date'. Participants included students, young innovators and founders of start-ups who competed with short pitches of their ideas or products. Winners attract the attention of those who are able to fund and turn the ideas into practical solutions that benefit society.

While it is difficult to gauge tangible outcomes from such a conference, there is little denying that participating in crossdisciplinary meetings offers invaluable experiences. It helps broaden horizons beyond the usual academic disciplines, encourages all to view the world through the lens of others, and obliges us to contribute to resolving the global challenges of the day. Scientists have a responsibility beyond the classroom and beyond exchanging technical knowledge between their academic circles. Scientists can empower those less fortunate and use their position to promote peace and add their voice to humanitarian laws (Nature 527, 7-8; 2015). While Joanne Liu, the International President of Médecins Sans Frontières, received a standing ovation at the end of her talk that called on world leaders to protect humanitarian spaces with the phrase "Hospitals must not be bombed", scientists must go beyond simple applause. Collectively, scientists can have a voice by petitioning to world leaders to rethink the indifference.

As emerging digital and physical technologies begin to converge, transforming the way products are made and used (a movement referred to as the fourth industrial revolution), multistakeholder dialogues that educate, and engage meaningfully and empathically must take place to ensure responsible progress, as outlined in the Thesis by Andrew Maynard on p1005. With the right strategies, the Falling Walls conference may become a useful platform for such engagements.

We celebrate the fact that nanotechnology is a multidisciplinary field, comfortable with collaboration, but nanotechnology scientists must continue to expand their circles, support social causes, and encourage others to do the same.

\section{When the dots are joined}

\section{We consider the potential of colloidal quantum dot solids for optoelectronic applications.}

Colloidal semiconductor quantum dots (QDs), also known as semiconductor nanocrystals, have attracted attention since their discovery just over 30 years ago, primarily because of their optical properties. The emission of sharp lines, similar to those of atoms, with a wavelength that can be tuned with slight changes in size and composition and the relatively low cost of synthesis makes QDs attractive for a range of optoelectronic as well as biomedical applications.

In this issue, we focus on a less explored aspect of QDs, that is, the electronic transport in three-dimensional arrangements of QDs, also known as QD solids. Just like a crystal consists of an ordered distribution of atoms, a QD solid is an ordered arrangement of QDs, which can be seen as artificial atoms, kept together by a variety of organic linkers. In a Review on page 1013, Cherie Kagan and Chris Murray explore the developments in the chemistry of QD solid assembly, which after several years has led to the observation of electron mobilities larger than $10 \mathrm{~cm}^{2} \mathrm{~V}^{-1} \mathrm{~s}^{-1}$, indicative of an electronic band structure similar to that of high-mobility crystals. These values make the potential use of QD solids in a range of optoelectronic applications very promising.

Based on this potential, we asked Maksym Kovalenko, Jeffrey Urban and Arto Nurmikko, to discuss in a series of Commentaries (pages 994, 997 and 1001), the realistic potential of realizing commercially viable devices for applications in photovoltaics, thermoelectrics and light emission. The overall picture that emerges is that although the results are certainly promising, whether QD solids will be competitive with other materials considered for the various applications will depend on further improvement in device efficiency and costs.

Whether the electronic transport in QD solids will one day be used in commercial devices remains an open question. However, the concept that we can fabricate materials based on QDs, with new properties that can, in principle, be tuned at will by just using chemistry to vary the size of the constituents, their composition and their distance is simply tantalizing, and this should continue fuelling research for both fundamental and technological interest. 